\title{
Hybrid modeling method of dust suppression efficiency for dry fog dust suppression process
}

\author{
Hailiang $\mathrm{Li}^{*}$, and Hongyang $\mathrm{Li}$ \\ Army Academy of Armored Forces, Changchun, China
}

\begin{abstract}
The original mechanism model of dust suppression efficiency for dry fog dust suppression could not guide the actual process. In order to accurately predict the dust suppression efficiency in the process, a new mechanism model was established by my analysis of the process. But there were some factors that I could not establish the model. So a hybrid model combining mechanism model and support vector machine (SVM) was proposed. Using the data of dry fog dust suppression oval process, the hybrid model was simulated. The simulation results show that the hybrid model can accurately predict the dry fog dust suppression.
\end{abstract}

Keywords: Hybrid model, Dry fog dust suppression, Dust suppression efficiency, SVM.

\section{Introduction}

With the development of dry fog dust suppression system, it has become a mature and effective dust suppression technology, which is widely used to control the dust of raw materials during the loading and unloading process of ore and rock crushing, screening, transportation and other workshops. At the same time, in order to further improve the efficiency of dry fog dust suppression system, it is necessary to study the model of dry fog dust suppression process. However, due to the characteristics of dry fog dust suppression process, complex principle and many influencing factors, it is difficult to accurately predict the dry fog dust efficiency.

There is little research on the dust removal suppression of dry fog dust process at home and abroad. In 2001, Zhang Xiaoyan designed the dust suppression system, and put forward the mathematical model of the relationship between dust suppression efficiency and atomization effect [1]. In 2006, Li Weikang studied the mathematical model of charged water mist system in coal preparation plant [2].

However, the above-mentioned mechanism models of dust suppression process are all realized under certain assumptions, which leads to the low accuracy of energy consumption model of the whole production process. The hybrid model combines the mathematical model based on mechanism with the black box model based on data, which not only fully considers the physical characteristics of the process, but also uses the relevant data information. In this paper, through the mechanism analysis of dry fog dust suppression

* Corresponding author: xnm543@163.com 
process, combined with support vector machine technology, a hybrid model of dust suppression efficiency in dry fog dust suppression process is established, which lays the foundation for improving the dust suppression efficiency of dry fog.

\section{Mechanism model of dry fog dust suppression process}

The principle part of dry fog dust suppression can be divided into two parts [3]. The first is the liquid atomization process, followed by the spray dust suppression process. The droplet atomization process is mainly broken into small particles by external force, and the particle size obtained by different crushing methods is also different. In this paper, an aerodynamic nozzle is used, which mainly uses compressed air to break the droplets, and the mechanical structure of the nozzle generates sound waves. The sound waves will further break the droplet particles, and the particle size reaches 10 microns. The process of spray dust suppression is mainly achieved by using the principle of heterogeneous nucleation and the principle of droplet catching dust. According to the principle of heterogeneous nucleation, with the continuous evaporation of water mist, the dry fog in the air will be rapidly saturated. Under certain conditions, the saturated water vapor will occur heterogeneous nucleation, that is, water vapor will gather on the surface of dust particles to form a droplet, and the droplet containing dust particles will collide with other droplets, and further increase to become "rain" landing on the ground. At the same time, according to the principle of droplet dust catching, when the dust laden airflow passes through the fog area where droplets gather, the dust particles are captured by inertial collision, interception, condensation and diffusion between dust particles and droplets. These droplets form large droplets and finally land on the ground. The two processes of heterogeneous nucleation and droplet dust collection are synchronous in dry fog dust suppression.

According to the research of Zhang Chi [4] and Qin Jun [5], the average diameter of the atomizing nozzle studied in this paper has a certain relationship with liquid pressure, gas pressure and gas-liquid pressure ratio, which can be expressed by equation (1).

$$
D_{w}=A \cdot R^{2}+B \cdot R+C
$$

Where, $R$ represents the ratio of air pressure to hydraulic pressure $R=\frac{p_{g}}{P_{w}}, P_{g}$ is system air pressure value, $P_{w}$ is system water pressure value, and $A, B$ and $C$ are parameters related to the mechanical structure of atomizing nozzle.

According to the research results of Heidenreich et al. [6], it can be seen that the condensation growth rate of fine particles is very fast under the condition of certain water vapor temperature and water saturation, and the condensation growth process can be basically completed.

When the dust laden air flow encounters a droplet, it will flow around. Therefore, not all dust can contact with the droplet. Only the dust near the center line can be caught by the droplet through collision or interception. The streamline through which the dust passes can form a flow pipe with cross-section diameter of. The cross-sectional diameter of the flow tube is lower than that of the spherical droplet, so the probability of dust contacting a single droplet is the ratio of the cross-sectional area of the flow tube to the cross-sectional area of the droplet, which is expressed in letter $E$.

$$
E=\frac{y^{2}}{D_{w}^{2}}
$$

The approximate estimate of $E$ is as follows: 


$$
E=\frac{y^{2}}{D_{w}^{2}}=0.266 \ln K+0.59
$$

In the equation, the dimensionless parameter $K$ is the dimensionless inertia parameter, also known as Stokes criterion, the calculation equation is as follows:

$$
K=\frac{V_{r} \rho D_{f}^{2}}{9 \mu_{p} D_{w}}
$$

In the equation, $\rho$ is the density of dust particles, $D_{f}$ is the diameter of dust particles, $\mu_{p}$ is the dynamic viscosity of air.

Assuming that the number of dust particles per unit volume in the dust airflow is $n$, then the dust catching rate of a single droplet is $\operatorname{En}_{r} \pi \frac{D_{w}^{2}}{4}$, where $V_{r}$ is the relative velocity of dust laden air flow and droplet.

Assuming that the droplets are spherical, the number of droplets generated per unit time is $W$ and passing through the dust collecting space is $\frac{6 W}{\pi D^{3}}$ at the water supply flow rate.

After a certain period of time $t_{B}$, the number of droplets in the space is $\frac{6 W t_{B}}{\pi D^{3}}$, assuming that the number of droplets in the space is basically the same during the whole dedusting process.

The fog flow and dust laden air flow move relative to each other at relative speed of $V_{r}$, and the fog flow is regarded as stationary. The distance of dust laden air flow into the fog flow in time $d t$ is $d x$ (see Figure 1).

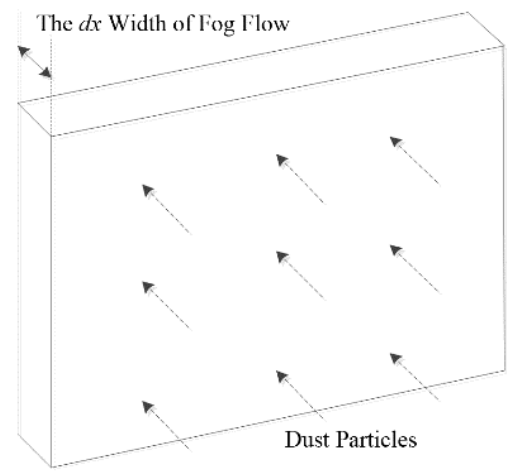

Fig. 1. Sketch map of fog flow dust collection.

When the dust laden air flows through the mist flow with width of $d x$, the dust concentration decreases from $n$ to $n-d n$, and the rate of dust concentration reduction is also the dust catching rate of the droplet group. The calculation equation of dust concentration reduction is as follows:

$$
\frac{3 E W n V_{r} t_{B}}{2 D_{w}}=\frac{d n}{d t}=\frac{d n}{d x} \cdot \frac{d x}{d t}=\frac{d n}{d x} V_{r}
$$

By integrating the equation along the length of the dust laden airflow passing through the mist flow, the reduction of dust concentration before and after the dust laden airflow passing through the fog flow with thickness of can be obtained as follows: 


$$
\int_{N_{w}}^{N_{w}} \frac{d n}{n}=\int_{0}^{L}-\frac{3 E W t_{B}}{2 D_{w}} d x
$$

In the equation, $N_{i n}$ is the dust number before the dust containing gas flows through the fog flow, and $N_{\text {ou }}$ is the dust number after the dust containing air flow passes through the fog flow.

The total dust collection efficiency of droplet group is as follows:

$$
\eta=1-\frac{N_{\text {out }}}{N_{\text {in }}}=1-\exp \left(-\frac{3 E W t_{B} L}{2 D_{w}}\right)
$$

According to fluid mechanics, the flow rate of fluid $W$ is related to pipe diameter, resistance and pressure difference between two ends as follows as follows:

$$
W=\mu_{g} S\left(\frac{2 P_{w}}{\rho}\right)^{1 / 2}
$$

where, $\mu_{\mathrm{g}}$ is the flow coefficient, which is related to the shape of the valve or pipe; $S$ is the cross-sectional area of the valve or pipeline; $P_{w}$ is the hydraulic pressure difference in the pipeline; $\rho$ is the liquid density.

By substituting equation (1), (3), (4) and (8) into equation (7), the mechanism model of the system can be obtained.

$$
\eta=1-\exp \left(-\frac{3 \mu_{g} S L P_{w}^{3} E t_{B}}{\left(2 P_{w} \rho\right)^{1 / 2}\left(A P_{g}^{2}+B P_{w} P_{g}+C P_{w}^{2}\right)}\right)
$$

According to the above theoretical analysis, it is known that the model of dry fog dedusting process is related to many factors, such as the particle size of the mist flow, the particle size of the dust, the preparation time of the pre spray, the space range of the dry fog and so on.

\section{Hybrid model of dry fog dust suppression process}

In this paper, a hybrid model combining mechanism model and SVM data model is established. The hybrid model not only reduces the requirements of mechanism model, but also makes full use of the existing data information, so as to realize the accurate prediction of dust suppression efficiency in dry fog process. The structure diagram of the hybrid model is shown in Figure. 2.

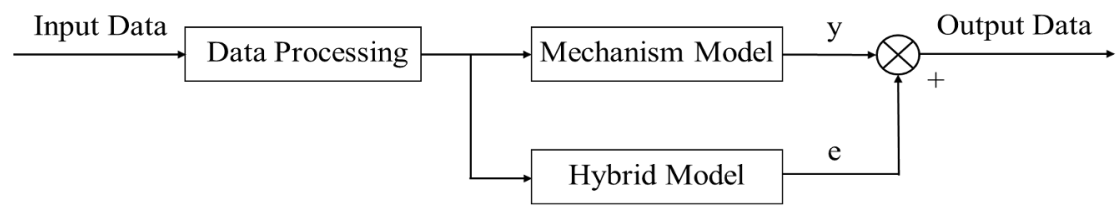

Fig. 2. Sketch map of fog flow dust collection.

Support vector machine regression (SVR) is an extension of support vector machine classification theory, which provides a new method for solving modeling problems. The linear regression estimation problem based on SVR can be transformed into the following optimization problems [7]- [8]: 


$$
\left\{\begin{array}{l}
\min \frac{1}{2}\|\omega\|^{2}+C_{s} \sum_{i=1}^{n}\left(\xi_{i}+\xi_{i}^{*}\right) \\
\text { s.t.y }\left\{\begin{array}{l}
y_{i}-\left(\omega \cdot x_{i}+b\right) \leq \xi_{i}+\varepsilon \\
\left(\omega \cdot x_{i}+b\right)-y_{i} \leq \xi_{i}^{*}+\varepsilon \\
\xi_{i}, \xi_{i} \geq 0, i=1,2, \ldots, n
\end{array}\right.
\end{array}\right.
$$

where $C_{s}$ is the penalty coefficient, $\varepsilon$ is the fitting accuracy, and $\xi_{i}$ is the lower limit and $\xi_{i}^{*}$ is the upper limit of the relaxation variable. According to the support vector classification problem, Lagrange function is also introduced into the regression problem. Finally, the linear regression function is obtained as follows:

$$
f(x)=\sum_{x \in S Y_{Y}}\left(\alpha_{i}-\alpha_{i}^{*}\right)\left(x \cdot x_{i}\right)+b
$$

where $\alpha_{i}, \alpha_{i}^{*}$ is the introduced Lagrange factor and $S V$ is the set of support vectors.

In order to solve the problem of linear indivisibility, kernel function is introduced to replace the point product in SVM to realize the mapping from input space to highdimensional space, in which linear discriminant function can be used to classify samples. The classification function is as follows:

$$
f(x)=\operatorname{sgn}\left(\sum_{i=1}^{n} \alpha_{i}^{*} y_{i} K\left(x \cdot x_{i}\right)+b^{*}\right)
$$

There are many kernel functions commonly used. The kernel function used in this paper is Gaussian kernel function:

$$
K\left(x \cdot x_{i}\right)=\exp \left(-\frac{\left\|x-x_{i}\right\|}{2 \sigma^{2}}\right)
$$

On the premise that the SVM model adopts Gaussian kernel function and the number of training samples is fixed, the generalization performance of SVM completely depends on the two parameters used in regression, namely penalty factor $C_{s}$ and kernel function parameter $\sigma$.

\subsection{Parameter selection based on SVM hybrid model}

The penalty factor in SVM model determines the degree of sample penalty for prediction error. The kernel function parameters implicitly determine the mapping function and feature space, and have an important impact on the performance of SVR. Therefore, the selection of optimal and combination becomes the key problem. In this paper, particle swarm optimization (PSO) is used to optimize the parameters of SVM.

In this paper, the actual dust suppression process in a mining area, after consulting the relevant references and materials, some relevant production parameters are selected or fitted as follows: $V_{r}=15 \mathrm{~m} / \mathrm{s}, \rho=1 \times 10^{3} \mathrm{Kg} / \mathrm{m}^{3}, D_{f}=5 \mu \mathrm{m}, S=0.008 \mathrm{~m}^{2}, t_{B}=600 \mathrm{~s}, L=2.5 \mathrm{~m}$, $\mu_{p}=1.8 \times 10^{-5}, \mu_{g}=0.6, A=3.13, B=-12.3, C=24.67 .220$ groups of effective data were collected, input as system air pressure $P_{g}$ and system water pressure $P_{w}$, and output as system dust suppression efficiency $\eta$. Among them, 180 groups of data are used as training samples, and 40 groups of data are used as test samples. After training, SVM parameters are finally selected as follows: $C_{s}=28.02, \sigma=26.35$. 


\subsection{Simulation verification}

The simulation results of the mechanism model and the hybrid model are shown in Figure. 3 and Figure. 4. In order to further verify that the hybrid model has better prediction performance, the results of the hybrid model proposed in this paper are compared with the simulation results of the mechanism model, as shown in Table 1.

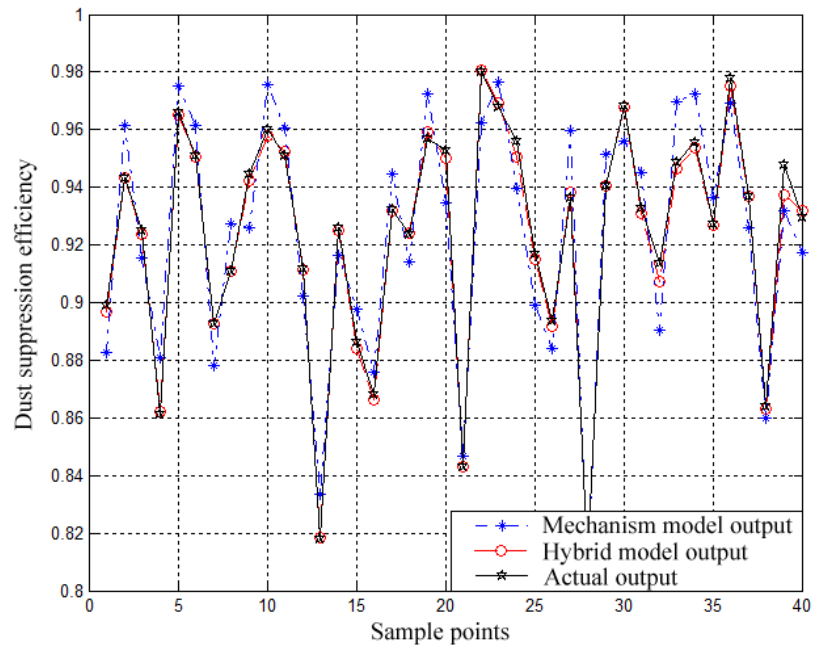

Fig. 3. Hybrid model output, mechanism model output and actual output contrast diagram.

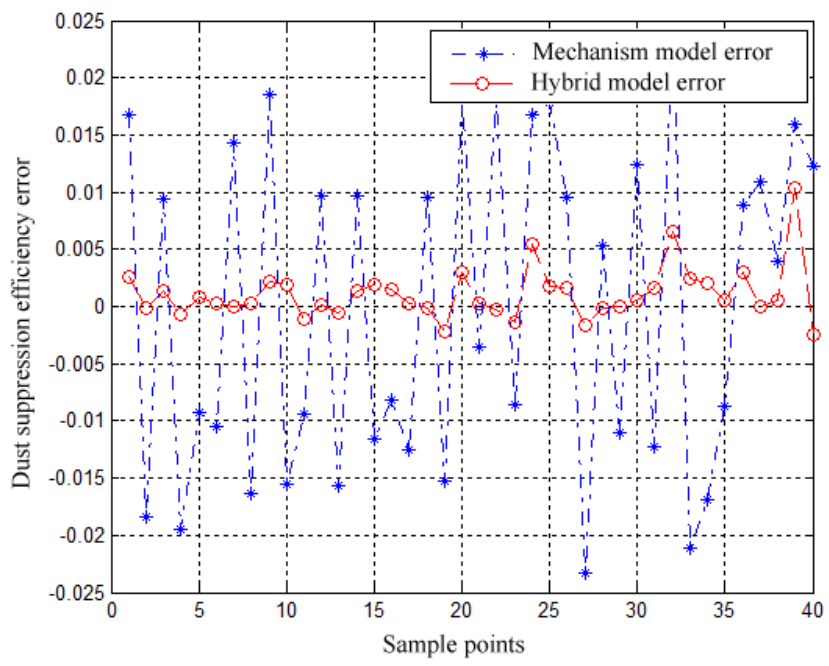

Fig. 4. Hybrid model and mechanism model output error curve.

Table 1. Performance evaluation of mechanistic and hybrid model-MAE, RMSE, MAPE, R.

\begin{tabular}{lcccc}
\hline & MAE & RMSE & MAPE $(\%)$ & R \\
\hline Mechanism model & 0.0132 & 0.0141 & 1.43 & 0.0132 \\
Hybrid model & 0.0056 & 0.0112 & 0.57 & 0.0056
\end{tabular}

From the above comparison results, it can be seen that the principle and structure of the mechanism model are correct. Due to the limitations of the mechanism model itself, the 
pure mechanism model can not describe all the characteristics of the process, and the parameters of the mechanism model obtained by data identification and literature review may not be accurate, so the error of mechanism model prediction is inevitable. Compared with the mechanism model, the hybrid model has better prediction effect and can meet the needs of dust suppression process prediction.

\section{Conclusion}

In this paper, through the analysis of the mechanism of oil dry fog dust suppression process, the mechanism model of dust suppression efficiency of dry fog dust suppression process is established. The error that cannot be described by the mechanism model is compensated in parallel by using SVM. Finally, the hybrid model of dust suppression efficiency in dry fog dust suppression process is obtained. Through simulation and comparison, it can be seen that the hybrid model can better estimate the dust suppression process of dry fog, and has a high performance. The prediction accuracy is an important theoretical basis for improving the efficiency of dry fog dust suppression.

\section{References}

1. Zhang Xiaoyan 2001 Design and experimental study of dusting system by fine water spay (Beijing: Industrial Safety and Environmental Protection) p 1-4

2. Li Weikang, Yang Xin. 2006 Research in Mathematics Model of Electrostatics Dust Removal System of Selecting Coal Works (Beijing: Coal Mine Machinery) p 43-45

3. Zhou WX and Yu ZH 2004 Multifractality of drop breakup in the air-blast nozzle atomization process (New York: Physical Review E Statistical Nonlinear \& Soft Matter Physics) p 175-181

4. Zhang Chi, Zhang Rongwei. 2006 Experimental investigation on atomization of an airblast atomizer with plain-orifice nozzle and dual-swirl cup (Beijing: Journal of Aerospace Power) p 805-809

5. Qin Jun, Chen Mozhi. 2005 Experimental study on Twin-Fluid Air-Blast Nozzle Atomization Under High Ambient pressures (Beijng: Journal of Combustion Science and Technology) p 384-387

6. Heidenreich S, Ebert F. Condensational droplet growth as a preconditioning technique for the separation of submicron particles from gases (New York: Chemical Engineering \& Processing) p 235-244

7. Vapnik V N 1998 Statistical learning theory (New York: John Wiley and Sons) p 1977

8. Kim K, Lee J M. 2005 A novel multivariate regression approach based on kernel partial least squares with orthogonal signal correction (New York: Chemometrics and Intelligent Laboratory Systems) p 79(2) 